\title{
Traduire
}

Une eutre perspective sur $r$ tatadciction

Revue française de la traduction

$242 \mid 2020$

Passons au vert

\section{La symbiose entre l'homme et la nature chez François Cheng}

\section{Guochuan Zhang}

\section{(2) OpenEdition}

1 Journals

Édition électronique

URL : http://journals.openedition.org/traduire/2053

DOI : 10.4000/traduire.2053

ISSN : 2272-9992

Éditeur

Société française des traducteurs

\section{Édition imprimée}

Date de publication : 30 juin 2020

Pagination : 126-138

ISSN : 0395-773X

\section{Référence électronique}

Guochuan Zhang, «La symbiose entre l'homme et la nature chez François Cheng », Traduire [En ligne], 242 | 2020, mis en ligne le 15 juillet 2020, consulté le 20 décembre 2020. URL : http:// journals.openedition.org/traduire/2053; DOI : https://doi.org/10.4000/traduire.2053 


\section{La symbiose entre I'homme et la nature chez François Cheng}

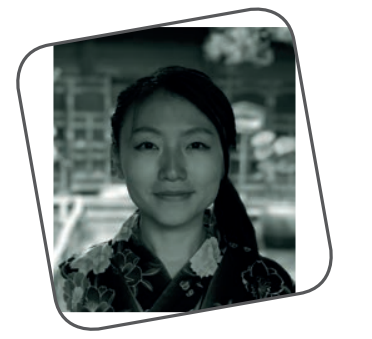

\section{Guochuan Zhang}

Dans cet article, nous nous emploierons à éclaircir la relation entre l'homme et la nature dans les traductions de François Cheng, académicien d'origine chinoise. Auteur de L'Écriture poétique chinoise, suivie d'une anthologie des poèmes des Tang (1977), il a traduit dans ce recueil 87 poèmes des Tang, ainsi que les théories picturales chinoises regroupées dans son livre intitulé Souffle-Esprit. Textes théoriques chinois sur l'art pictural. Ses traductions transmettent la vision du monde des lettrés chinois et mettent en lumière la continuité du dialogue entre l'homme et la nature dans la tradition chinoise.

En nous basant sur ses traductions de poèmes et de théories, nous essaierons de clarifier son concept d'attachement de l'homme à la nature, qui évolue vers leur symbiose sous la triple influence des pensées bouddhiste, taoïste et chrétienne.

L'intimité entre l'homme et la nature qui marque l'art chinois est déjà présente dans la mythologie de la naissance du monde. Avant d'aborder la poésie chinoise, observons 
d'abord le mythe chinois de la création du monde par un dieu nommé Pangu:

Le ciel et la terre formaient un ensemble pareil à un œuf de poule. Pangu naquit en son milieu. Au bout de dix-huit mille ans, le ciel et la terre se séparèrent. Le yang pur constitua le ciel et le yin grossier la terre. Pangu se trouvait en leur milieu. Lorsqu'il fut sur le point de mourir, il transforma son corps: son souffle devint les vents et les nuées, sa voix les éclats du tonnerre, son œil gauche le soleil, son œil droit la lune, ses quatre membres et les cina [parties de son] corps les quatre extrêmes et les cinq montagnes sacrées, son sang et ses humeurs le fleuve Bleu et le fleuve Jaune, ses nerfs et ses artères les veines de la terre, ses muscles la glèbe des champs, ses cheveux et ses moustaches les astres et les repères sidéraux, les poils de sa peau la végétation, ses dents et ses os les métaux et les pierres, ses essences et sa moelle les perles et les jades, sa sueur et ses écoulements les pluies et les marais'.

Pangu s'incarne dans tous les éléments végétaux et minéraux, et le monde est ainsi créé. L'art chinois, puisant sa première inspiration dans la mythologie, traduit cet attachement de l'homme au cosmos. Les poètes ont souvent recours aux éléments de l'environnement, omniprésents dans la poésie classique. Dans leurs œuvres, ces images sont toujours suggestives et imprégnées d'émotions humaines. Dans l'anthologie la plus ancienne rassemblant les poèmes de l'Antiquité chinoise datant $d u \times l^{e}$ siècle av. J.-C., Shijing (Livre des odes), les éléments de la nature rappellent déjà les émotions personnelles des poètes. Deux figures de style y sont le plus souvent utilisées: le bi (comparaison) et le xing (incitation). Dans L'Écriture poétique chinoise, François Cheng explique ainsi ces deux procédés:

Le bi (comparaison) est employé lorsque le poète fait appel à une image (de la nature en général) pour figurer une idée ou un sentiment qu'il voudrait exprimer. On use en revanche du xing (incitation) quand un élément du monde sensible, un paysage

1. Rémi Mathieu (trad.), Anthologie des mythes et légendes de la Chine ancienne, Paris, Gallimard, 1989, p. 28. 
ou une scène, suscite chez lui un souvenir, un sentiment latent ou une idée jusque-là non exprimés².

Il voit à travers ces deux figures de style un rapport toujours renouvelé entre l'humain et l'univers, car «le bi incarne le processus sujet-objet, celui qui va de l'homme vers la nature, tandis que le xing introduit le processus inverse objet-sujet, celui qui part de la nature pour revenir à l'homme $e^{3}$ ».

Afin d'éclaircir cette intimité entre l'univers et les poètes chinois, arrêtons-nous sur l'image des saules dans un quatrain de Wang Changling (698-757) traduit par François Cheng, intitulé Complainte du palais:

Jeune femme en son gynécée ignorant les chagrins Jour de printemps, parée, elle monte sur la tour Éblovie par la teinte des saules le long du chemin Regret soudain: de son époux parti chercher les honneurs ${ }^{4}$

Ici, les saules, rappelant à la jeune femme le jour de sa séparation avec son époux, portent deux connotations principales. Tout d'abord, comme François Cheng l'écrit, «le saule, par sa couleur tendre et ses branches gracieuses, symbolise le printemps et la jeunesse ${ }^{5}$ ». La jeune femme s'aperçoit soudain de l'écoulement irréversible du temps. La douleur de la séparation et le regret de sa jeunesse éphémère se mêlent dans cette complainte. Par ailleurs, dans la langue chinoise, le mot 《saule» (柳/iu) est homonyme du mot «rester» (留liu). Ainsi, les Chinois anciens en offrent souvent une branche à un ami ou à un amant qui part, ceci afin d'exprimer leur peine causée par la séparation. Cette plante est ainsi devenue une image récurrente qui incarne la nostalgie depuis la première anthologie de la poésie chinoise jusqu'à nos jours.

Cette image nous permet d'avoir un aperçu de la vision du monde des lettrés chinois qui se considèrent comme les confidents intimes de la nature. François Cheng nous transmet cette vision du monde par ses traductions des poèmes classiques des Tang et par celles des essais théoriques picturaux. Pour Zhang Yinde, l'ouvrage de François Cheng

2. François Cheng, L'Écriture poétique chinoise, Paris, Éditions du Seuil, 1996, p. 92.

3. Ibid., p. 93.

4. Ibid., p. 150.

5. Idem. 
intitulé D'où jaillit le chant. La Voie des Fleurs et des Oiseaux dans la tradition des Song met au centre le paysage qui jouit «du privilège de représenter cet art millénaire». "Fleurs et oiseaux», par leur sujet d'apparence humble et familière, permettent à François Cheng de sonder, "dans le sillage des artistes depuis les Song, le secret de la nature et le mystère existentiel des êtres et des choses ${ }^{6} \gg$.

Afin d'examiner l'intimité entre l'homme et la nature qui se manifeste dans les théories picturales chinoises traduites par François Cheng, nous nous concentrerons sur deux images: les rochers et les arbres. Les peintures classiques chinoises à l'encre se répartissent en trois grands genres: "montagne et eau», "fleurs et oiseaux» et "portraits». En tant que thème principal des deux grands genres picturaux, les arbres et les rochers sont liés par une harmonie et une dépendance mutuelle appréciées des lettrés chinois. Dans Souffle-Esprit, consacrant un chapitre entier intitulé Arbres et rochers aux théories picturales chinoises sur la composition des arbres et des rochers dans les peintures de "montagne et eau», François Cheng explicite leurs valeurs philosophiques et la technique de dessin. Les deux images composent souvent un ensemble: rocher stérile avec arbre maigre, rocher fertile avec arbre luxuriant. "Étant un "descendant" de la montagne, le rocher a pour "conjoint" l'arbre. Sans arbre, un rocher est privé d'abri; sans rocher, un arbre, lui, est privé d'appui ${ }^{7} . »$ Dans l'esthétique traditionnelle chinoise, les montagnes doivent être présentées sous des formes variées, comme les arbres. Ceux-ci forment des vêtements pour les montagnes et ces dernières constituent pour les arbres une ossature. Ces deux images de la nature composent un tout dans la peinture classique chinoise: le manque de l'un nuirait à l'harmonie de la beauté picturale.

Dans ce livre, François Cheng évoque la théorie de Wang Yuanqi, peintre des Qing: selon les Anciens, les essences du ciel et de la terre s'unissent pour former les rochers.

6. Yinde Zhang, "François Cheng ou dire la Chine en français», in Revue de littérature comparée, $n^{\circ} 322,2007$, p. 151.

7. François Cheng, Souffle-Esprit. Textes théoriques chinois sur l'art pictural, Paris, Éditions du Sevil, 1989, p. 63. 
Ces derniers représentent la concentration du souffle. Les rochers d'où naissent les nuages portent ainsi le nom de «racines des nuages». Dans le Poème composé pour l'ermitage de Li Ning traduit par François Cheng, nous lisons le vers suivant:

Le pont traversé, un paysage autre s'ouvre devant soi Déplaçant le rocher, on libère les racines des nuages ${ }^{8}$

Cette représentation mythique et taoïste des rochers traduit l'attachement des lettrés à cet élément de la nature.

Les rochers sont appréciés par les artistes chinois pour leurs qualités: la solidité, la persistance, l'incarnation de l'éternité. Leur pérennité fait contrepoint à l'éphémère humain. Malgré l'écoulement du temps, ils restent inchangés. Silencieux et immobiles, ils refusent tout déguisement. Les pierres «détentrices du souffle de l'initiale ${ }^{9} \gg$ gardent intacts tous les tourments et les joies de la vie terrestre. L'homme découvre une partie de lui-même grâce à ces objets de l'univers. Un dialogue est tissé entre l'homme et la nature. L'existence de l'«Autre» représentée ici par les pierres et les rochers aide les humains à reconnaître leur propre existence.

Les pierres et les rochers incarnent l'univers originel, alors que les arbres reflètent le devenir humain: la naissance, la croissance, la luxuriance et la flétrissure. Les racines, les troncs, les rameaux et les feuilles formant un cercle de vie, de concert, ils tendent vers le haut et aspirent à l'infini. Cette aptitude à devenir est mise en contraste avec celle d'éternité des rochers. Ensemble, les deux éléments de la nature nous communiquent la règle commune de l'univers: le Tao.

Dans la culture traditionnelle chinoise, nous apercevons l'importance des arbres pour les artistes. Ils surnomment Trois amis partemps froid le pin, le bambou et le prunus, car ces trois arbres gardent leur verdure durant l'hiver, alors que

8. Ce poème de Jia Dao (贾岛779-843) a été traduit par François Cheng dans Entre source et nuage, Paris, Albin Michel, 2002, p. 91. Voici le poème entier: "Demeure oisive environnée de rares voisins / Le sentier herbeux pénètre un jardin négligé / Sur les arbres de l'étang, les oiseaux se nichent en confiance / Sous la lune, à la porte, un moine discrètement frappe / Le pont traversé, un paysage autre s'ouvre devant soi / Déplaçant le rocher, on libère les racines des nuages / Sur le point de partir, déjà je songe à revenir / À la date promise, je serai au rendez-vous.»

9. François Cheng, À l'orient de tout, Paris, Gallimard, 2005, p. 19. 
les Quatre hommes de bien représentent le prunus, l'orchidée, le bambou et le chrysanthème. Fleurissant à la fin de l'hiver et au début du printemps, le prunus symbolise l'orgueil et la noble aspiration. Comme l'orchidée, il fait souvent référence aux ermites et évoque la beauté isolée. À feuillage persistant, le pin et le bambou incarnent l'homme accompli, ferme et droit. Ce n'est d'ailleurs pas un hasard si l'épée de François Cheng l'académicien, créée par le joaillier Mellerio, porte une branche de bambou.

Dans Vide et plein, le langage pictural chinois, François Cheng transmet aux lecteurs francophones l'attachement aux arbres des artistes chinois. Selon lui, ces derniers ont fréquemment recours à ces éléments végétaux afin d'exprimer leurs propres valeurs et attentes. Sous le pinceau de Shitao, ils sont dotés de caractères humains:

Ma méthode pour peindre les pins, les cèdres, les vieux acacias et les vieux genév riers est de les grouper par exemple par trois ou cinq, en combinant leurs attitudes: certains se dressent d'un élan hérö̈que et guerrier, certains baissent la tête, d'autres la relèvent, tantôt ramassés sur eux-mêmes, tantôt campés bien droits, ondulants ou balancés ${ }^{10}$.

Plantés profondément dans le sol, ces plantes symbolisent les êtres humains vivant dans ce monde. Ils puisent leur force dans la terre et essaient de s'élever. Pourtant, ces plantes restent entravées et clovées: leur douleur est infinie.

Les arbres de l'infinie douleur

Les nuages de l'infinie joie

Se donnent parfois signe de vie

À la lisière du vaste étén

Ces vers de François Cheng inspirés par l'art chinois nous rappellent la première vérité du Bouddha: lors de son premier enseignement, le Bouddha affirme que si l'on observe attentivement et honnêtement la vie humaine, on ne peut que constater que celle-ci est remplie de souffrances. À travers les arbres, François Cheng nous communique son

10. François Cheng, Vide et plein. Le langage pictural chinois, Paris, Éditions du Seuil, 1991, p. 133.

11. François Cheng, À l'orient de tout, op. cit., p. 70. 
étonnement vis-à-vis du contraste entre la beauté naturelle et la misère humaine.

Comme Wang Wei, poète-peintre des Tang qui constitue pour lui une grande source d'inspiration. François Cheng fait partie des lettrés chinois attachés au bouddhisme et au taoïsme. Une image, en particulier, met en lumière l'influence de ces deux pensées chez lui et chez Wang Wei: la "montagne vide». Examinons une strophe de François Cheng:

Pommes de pin tombées

Dans la montagne vide

Tu les entends n'est-ce pas

Là où tu es

En lieu séparé

Mais au même instant ${ }^{12}$

Ces vers nous rappellent, par leur tonalité, un poème de Wang Wei, traduit par François Cheng dans L'Écriture poétique chinoise:

Repos de l'homme. Chute des fleurs du cannelier

Nuit calme, de mars, dans la montagne déserte

Surgit la lune; effrayé, l'oiseau crie.

Échos des cascades printanières...13

Chez ces deux poètes, la «montagne vide» pourrait être interprétée de deux façons, bouddhique et taoïste. Pour les bouddhistes, ce qui est Forme est Vide, ce qui est Vide est Forme. Cette montagne vide pourrait laisser supposer que le poète perçoit que la forme ne diffère pas du vide, et que le vide ne diffère pas de la forme: le poète est ainsi libéré de toute souffrance terrestre. Pour les taoïstes, "trente rayons convergent au moyeu, mais c'est le vide médian qui confère à la voiture sa fonction. On façonne l'argile pour faire des vases, mais c'est du vide interne que dépend son usage. Une maison est percée de portes et de fenêtres, c'est encore le vide qui permet l'usage de la maison ${ }^{14}{ }^{\prime}$. Dans les poèmes de Wang Wei et dans ceux de François Cheng, les images

12. Ibid., p. 253.

13. François Cheng, L'Écriture poétique chinoise, op. cit., p. 139.

14. Kia-Hway Liou et Benedykt Grynpas (trad.), Philosophes taoïstes, Lao-tseu, Tchouangtseu, Lie-tseu, Paris, Gallimard, Bibliothèque de la pléiade, 1993, p. 13. 
sont toujours associées par leurs aspects opposés: I'homme vivant et les fleurs fanées, l'oiseau dynamique et la lune immuable, les cris et les ravines silencieuses. "L'être et le néant s'engendrent l'un l'autre. Le facile et le difficile se parfont. Le long et le court se forment l'un par l'autre. Le haut et le bas se touchent. La voix et le son s'harmonisent. L'avant et l'après se suivent ${ }^{15}$.»Dans ce sens, la "montagne vide» contenant le dynamisme de la nature est à l'origine de toute plénitude.

L'intimité entre l'humain et l'univers dans l'art chinois ne s'arrête pas à un simple attachement: ces deux entités constituent une symbiose vivante. "Pour les Chinois, un existant est aussi bien une plante, un rocher, un arbre, un nuage, un animal, qu'un être humain, dont l'artiste. L'homme n'est qu'un existant parmi les autres ${ }^{16}$. $"$ Dans la tradition chinoise, les lettrés rendent de temps à autre visite à un ermite qui habite au sommet d'une montagne. Dans ce lieu loin du terrestre humain, l'ermite vit pleinement la présence de la nature et de son propre esprit, communiant ainsi en profondeur avec le cosmos. Un quatrain de Jia Dao, traduit par François Cheng, illustre cette symbiose:

Sous le pin, j'interroge le disciple.

"Le maitre est parti chercher des simples,

Par là, au fond de cette montagne.

Nuages épais: on ne sait plus où... $\rangle^{17}$

Souvenons-nous de l'histoire de Zhuangzi, grand penseur taoïste: "Jadis, Zhuangzi rêva qu'il était un papillon voltigeant et satisfait de son sort et ignorant qu'il était Zhuangzi lui-même. Brusquement, il s'éveilla et s'aperçut avec étonnement qu'il était Zhuangzi. Il ne sut plus s'il était Zhuangzi rêvant qu'il était un papillon, ou un papillon rêvant qu'il était Zhuangzi ${ }^{18}$.» Zhuangzi et le papillon ne font qu'un. Dans ses propres poèmes, François Cheng s'assimile lui aussi aux fourmis, aux moineaux et au vieux chêne. Les artistes chinois,

\footnotetext{
15. Ibid., p. 4.

16. Yolaine Escande, Philippe Sers, Résonance intérieure, dialogue sur l'expérience asiatique et sur l'expérience spirituelle en Chine et en Occident, Paris, Klincksieck, 2003, p. 18.

17. François Cheng, Entre source et nuage, op. cit., p. 90.

18. Kia-Hway Liou et Benedykt Grynpas, Philosophes taoïstes, op. cit., p. 104.
} 
disciples éternels du Tao, entrent en communion avec la nature et y perçoivent le vrai, le beau, le véritable sens de l'art. La nature est une représentation du concept cosmologique chinois: la montagne symbolise le souffle yang, l'eau le souffle yin, et le nuage le vide médian, invisible et immuable, où s'opère la métamorphose de toutes choses, y compris de I'homme.

De Laozi à nos jours, l'esthétique chinoise a toujours mis l'accent sur la beauté naturelle. Les meilleurs objets d'art, comme les poèmes les plus exquis, sont dépourvus de trace artificielle et restent naturels comme les rochers non sculptés, incarnation de la beauté irrégulière qui instille le rythme de l'univers. Ce retour à la pureté, au dépouillement et à la nature incarne non seulement une notion clé du taoïsme, mais aussi une notion chrétienne, incarnée par saint François d'Assise, auteur du Cantique des créatures.

Dans les notions chrétiennes, la symbiose de l'homme et de la nature tient également une place importante. En 1961, lors d'un voyage à Assise, François Cheng découvre saint François d'Assise, qu'il qualifie de «Grand vivant». Une fois arrivé dans cette blanche cité perchée sur la colline, il ressent l'omniprésence du saint: "Chaque herbe, chaque fleur, chaque insecte, chaque oiseau témoigne de lui ${ }^{19}$. $\gg$ Aux Carceri, au sein de cet univers de grottes habité par le saint, François Cheng se sent «de connivence avec lui dans l'amour de la pierre». Il le voit "à la manière de tant d'ermites taoïstes, dormir au creux des rochers avec, en guise d'oreiller, un gros caillou à la surface lisse». Si François Cheng a choisi, lors de sa naturalisation en 1971, le prénom «François» en hommage au saint, c'est qu'il partage avec ce frère universel cette communion avec la nature, illustrée, d'une part, par le Cantique des créatures de saint François d'Assise, et, d'autre part, par les titres des recueils de poèmes chengiens où la nature est omniprésente, tels De l'arbre et du rocher et Cantos toscans. De plus, ils aspirent tous les deux à l'idéal du retour à la pureté et au dépouillement, commun au taoïsme et au christianisme.

La pierre constitue un élément faisant écho entre François Cheng et saint François d'Assise, alors que l'arbre est un

19. François Cheng, Assise, une rencontre inattendue, Paris, Albin Michel, 2014, p. 21. 
thème commun pour François Cheng et Paul Claudel, l'écrivain de foi chrétienne. Pour l'auteur de J'aime la Bible, l'arbre incarne le cercle de l'univers: "L'arbre est l'œuvre, l'expression et la mesure du temps dont il inscrit dans son tronc les périodes successives en cercles concentriques. Chaque année, par le moyen de la fleur, il demande au ciel un fruit. Le fruit qui sous une enveloppe est nourriture et semence. Après quoi l'arbre meurt et il est livré aux flammes ${ }^{20}$.» Une allusion à la Bible se dégage de cet extrait. En fait, si ce motif est tellement cher à Claudel, c'est qu'il renvoie à l'arbre du Jardin d'Éden et à l'arbre de la Croix. II est "essentiel dans toute l'œuvre claudélienne où il symbolise l'homme et incarne le lien entre verticalité et horizontalité, terre et ciel, concret et spiritue|${ }^{21} \gg$. Parallèlement, en lisant Vraie lumière née de vraie nuit de François Cheng, Pierre Brunel, spécialiste de Paul Claudel, constate chez lui une référence à la Bible, plus précisément au «vieil arbre de la Genèse - Arbre de vie il est vrai, dont trop tôt, trop imprudemment, Adam et Ève ont voulu goûter le fruit»: "Vieil arbre / Muré dans son mutisme / Depuis si longtemps ${ }^{22}$. Ce recueil de poèmes est d'ailleurs pour Pierre Brunel inspiré par la Bible, dont le titre fait écho à un verset de la Genèse:

Dieu dit: vienne la lumière./ Et la lumière fut./ Dieu voit la lumière. elle est bonne. / Et Dieu sépare la lumière et la ténèbre. / Dieu nomme la lumière Jour et la ténèbre Nuit. I II est un soir, il est un matin: premier jour ${ }^{23}$.

Par ses traductions de poèmes et de théories, François Cheng nous transmet la symbiose vivante entre l'humain et le paysage. Par ses propres créations poétiques et artistiques, le poète détient, comme tous les artistes, un autre statut de «traducteur»: celui de traducteur de la beauté du cosmos.

La lecture des livres occidentaux, tels que Les Nourritures terrestres d'André Gide, favorise chez François Cheng son

20. Paul Claudel, J'aime la Bible, Paris, Ecclesia, 1955, p. 23.

21. Pascale Alexandre, présentation du recueil de Paul Claudel intitulé L'Arbre, www.paulclaudel.net/oeuvre/arbre, consulté le 29/05/2020.

22. Pierre Brunel, «Vraie lumière née de vraie nuit», dans François Cheng. À la croisée de la Chine et de l'Occident, Genève, Droz, 2014, p. 96.

23. La Bible, version, édition, Genèse 1. 
attachement pour la nature. Les peintres occidentaux, aussi proches du paysage que les artistes chinois, constituent également pour lui une source d'inspiration. Les représentations de la montagne Sainte-Victoire par Cézanne apparaissent à François Cheng comme un parangon de «rencontre entre l'esprit de l'homme et celui du paysage»:

Chez Cézanne, la beauté est formée de rencontres à tous les niveaux. Au niveau de la nature représentée, c'est la rencontre entre le caché et le manifesté, entre le mouvant et la fixité; au niveau de l'agir de l'artiste, c'est la rencontre entre les touches apposées, entre les couleurs appliquées. Et au-dessus de cet ensemble, il y a la rencontre décisive entre l'esprit de l'homme et celui du paysage 24 .

C'est grâce à l'art que l'humanité a préservé le beau. Grâce aux artistes, "le fil d'or du beau ne s'est [jamais] tout à fait interrompu». La nature n'est pas «une entité inerte et passive»: "Si l'homme la regarde, elle le regarde aussi; si I'homme lui parle, elle lui parle aussi ${ }^{25}$.» Un «renversement de perspective» s'effectue entre l'homme et l'univers: "Tandis que l'homme devient l'intérieur du paysage, celui-ci devient le paysage intérieur de l'homme ${ }^{26}$.»

La nature détient les secrets du monde. Ce qui est dit est donné à ses «traducteurs», les artistes: «le monde et son mot de passe ${ }^{27}$ ». Elle permet aux poètes de ressentir des émotions et de reproduire la beauté dans leur art. Pour Stéphane Mallarmé, "l'explication orphique de la terre [...] est le seul devoir du poète et le jeu littéraire par excellence ${ }^{28} »$. La mission du poète et du peintre consiste à «sonder le mystère de cet incessant échange»entre toutes les entités vivantes de cet univers, animée par le vide médian, et à «traquer ce qui, dans ce mouvement circulaire, se passe entre ${ }^{29}$. La pensée

24. François Cheng, Cinq méditations sur la beauté, Paris, Albin Michel, 2012, p. 134.

25. Ibid., p. 120.

26. Ibid., p. 103.

27. François Cheng, À l'orient de tout, op. cit., p. 149.

28. Stéphane Mallarmé, «À Paul Verlaine, Paris, 16 novembre 1885 », Correspondances [1872-1898], Paris, Folio classique, 1995, p. 583.

29. Madeleine Bertaud, "François Cheng, poète français», in Revue de littérature comparée, $n^{\circ} 322,2007$, p. 159. 
chinoise est ternaire, elle privilégie ce vide médian, ce lieu d'échange entre-deux.

Son idéal est d'instaurer le grand dialogue entre l'homme et la création (c'est la vision du Tao), et entre les hommes (pour la vision confucéenne). C'est aussi un principe de vie. En pratiquant le tai-chi-chuan, la gymnastique chinoise, le Chinois a la naïveté de croire qu'il suscite un souffle, le même que celui qui anime l'Univers. Un calligraphe suscite lui aussi un souffle ${ }^{30}$.

Dans l'art, ce vide médian est incarné par le trait de pinceau qui relie l'humain à la création. Shitao affirme qu'il détient le nœud de la montagne, son cœur bat en lui: "L'esprit du Paysage et mon esprit se sont rencontrés et par là transformés, en sorte que le Paysage est bien en moi ${ }^{31}$.» De même, le poète Su Shi préconise: "Avant de dessiner un bambou, laissez-le croitre d'abord en vous.» La symbiose entre l'homme et la nature, expliquée en détail dans ses traductions, constitue un concept majeur de la poésie de François Cheng. Cette vision du monde des lettrés de l'Antiquité chinoise nous inspire dans ce monde $\left.d u x x\right|^{e}$ siècle.

\section{karine_guochuan_zhang@yahoo.fr}

Titulaire d'une thèse intitulée La symbiose de la culture occidentale et de la culture chinoise dans la poésie de François Cheng, Guochuan Zhang dispense des cours de langue et de littérature chinoises dans des établissements secondaires depuis 2014. Ses travaux de recherche portent sur la littérature comparée entre la Chine et la France, notamment sur la coexistence des deux cultures dans la poésie de François Cheng, et sur l'influence chinoise dans les écrits de Victor Segalen.

30. «La peinture est la religion des Chinois», interview avec François Cheng, par Simonnet Dominique et Monier Françoise, L'Express, 15/10/1998.

31. François Cheng, Souffle-Esprit. Textes théoriques chinois sur l'art pictural, op. cit., p. 36. 


\section{SOURCES CITÉES}

BERTAUD Madeleine, "François Cheng, poète français ", in Revue de littérature comparée, $\mathrm{n}^{\circ} 322$, 2007, p. 153-164.

BRUNEL Pierre, «Vraie lumière née de vraie nuit», in François Cheng, À la croisée de la Chine et de l'Occident, Genève, Droz, 2014.

CHENG François, Vide et plein, langage pictural chinois, Paris, Éditions du Seuil, 1991.

CHENG François, Souffle-Esprit. Textes théoriques chinois sur l'art pictural, Paris, Éditions du Seuil, 1989.

CHENG François, L'Écriture poétique chinoise, Paris, Éditions du Seuil, 1996.

CHENG François, Entre source et nuage, Paris, Albin Michel, 2002.

CHENG François, Le Livre du vide médian, Paris, Albin Michel, 2004.

CHENG François, À l'orient de tout, Paris, Gallimard, 2005.

CHENG François, Cinq méditations sur la beauté, Paris, Albin Michel, 2012.

CHENG François, Assise, une rencontre inattendue, Paris, Albin Michel, 2014.

ESCANDE Yolaine, SERS Philippe, Résonance intérieure, dialogue sur l'expérience asiatique et sur l'expérience spirituelle en Chine et en Occident, Paris, Klincksieck, 2003.

LIOU Kia-Hway et GRYNPAS Benedykt (trad.), Philosophes taoïstes, Lao-tseu, Tchouang-tseu, Lie-tseu, Paris, Gallimard, «Bibliothèque de la pléiade», 1993.

MALlARMÉ Stéphane, "À Paul Verlaine, Paris, 16 novembre 1885», in Correspondances [1872-1898], Paris, «Folio classique», 1995.

MATHIEU Rémi (trad.), Anthologie des mythes et légendes de la Chine ancienne, Paris, Gallimard, 1989.

ZHANG Yinde, «François Cheng ou dire la Chine en français », in Revue de littérature comparée, n³22, 2007, p. 141-152. 